\title{
Semantic Commitment for Designing Ontologies: A Proposal
}

\author{
Bruno Bachimont ${ }^{1}$, Antoine Isaac $^{1,2}$, Raphaël Troncy ${ }^{1,3}$ \\ 1 Institut National de l'Audiovisuel, Direction de la Recherche \\ 4, Av. de l'Europe - 94366 Bry-sur-Marne \\ \{bbachimont, aisaac,rtroncy\}@ina.fr \& http://www.ina.fr/ \\ 2 LaLICC, Université de Paris-Sorbonne, http://www.lalic.paris4.sorbonne.fr \\ 3 INRIA Rhône-Alpes, Action EXMO, http://www.inrialpes.fr/exmo
}

\begin{abstract}
The French institute INA is interested in ontologies in order to describe the content of audiovisual documents. Methodologies and tools for building such objects exist, but few propose complete guidelines to help the user to organize the key components of ontologies: subsumption hierarchies. This article proposes to use a methodology introducing a clear semantic commitment to normalize the meaning of the concepts. We have implemented this methodology in an editor, DOE, complementary to other existing tools, and used it to develop several ontologies.
\end{abstract}

\section{Introduction}

With the emergence of technical systems which exploit numerical contents, accessing and processing information are evolving at a fair rate. The French institute INA $^{4}$ has to manage large multimedia and audio-visual databases, a task that includes allowing an access as efficient as possible to the data stored. INA is thus greatly concerned with indexing - the core of its mission -, which implies dealing with ontologies to create relevant content description of the audio-visual documents.

While trying to use ontologies, one soon has to face the problem of the way they are designed, especially in regard to taxonomy structuration. Indeed, it is acknowledged that the taxonomies of domain concepts are key components of the built ontologies. Consequently, we searched for a methodological approach that would give guidelines to structure taxonomies (Section 2). We claim that none of these methodologies force the ontologist to explicit the real meanings of the concepts and consider thereafter a possible solution, using natural language. We detail the three steps of a methodology proposal (Section 3) and present a tool implementing it, before concluding (Section 4).

\footnotetext{
${ }^{4}$ INA (Institut National de l'Audiovisuel) has been archiving TV documents for 45 years and radio documents for 60 years. It stores more than 700000 hours of broadcast programs (3000 000 audio-visual documents) and some 2000000 images.
} 


\section{Which Methodology for Building Ontologies?}

\subsection{A Work Still in Progress}

Many approaches (for a complete survey, the reader can refer to the OntoWeb Technical RoadMap ${ }^{5}$ ) have been reported to build ontologies, but few fully detail the steps needed to obtain and structure the taxonomies. For instance, METHONTOLOGY, proposed by the LAI of Madrid [2], is rather interested in giving methodological outlines for the whole process of ontology engineering. It focuses on the life cycle and the ordering of the general steps to develop these ontologies: identify the purpose of the information system, collect the relevant information for knowledge acquisition, evaluate the results, etc. Obviously, all these tasks are essential according to an "ontological engineering" point of view. However, the conceptualization step, in which the concepts and the relations between them are captured, has to be detailed. For example, Methontology proposes to build the ontology at the knowledge level using a set of intermediate representations. Although the taxonomy is one of these representations, the methodology does not stress on the way to classify the concepts in it.

\subsection{Requirements for a Methodology Focusing on Natural Language}

We claim that none of the existing methodologies force the ontologist to explicit the real meaning of the concepts in the most natural way: using natural language (NL). Actually, some methodologies recommend using NL to explicit the meaning of the concepts inside comments or through documents surrounding the modeling process, but not in a principled way. The terms used to denote the concepts are still liable to multiple interpretations. This results in possible misunderstandings and consequently bad modeling and use of the ontology. As a solution, we suggest to follow an evolved version of methodological guidelines that were first outlined in [1].

The first problem to face is the under-determination of meaning: every expression in language has its meaning contextually defined, since interpretation may vary according to the context (a specific application). Modeling will thus consist in choosing linguistic labels and associating with them a relevant and non-contextual semantics. The problem is then to determine which kind of semantics and how to use it in a normalization effort.

Second, defining a linguistic meaning is not sufficient to specify a system. A usual approach consists in associating a formal semantics with concepts. Formal semantics allows a mathematical modeling of the linguistic meaning as well as of the system behavior. The ontologist needs a semantics formal enough to efficiently specify computations, and yet close enough to the knowledge level to make these computations intelligible.

Finally, an ontology has to introduce knowledge primitives which will be the building blocks for programming a Knowledge-Based System (KBS). From this

\footnotetext{
${ }^{5}$ http://babage.dia.fi.upm.es/ontoweb/wp1/0ntoRoadMap/index.html
} 
point of view, a label will be used in rules, or grammars, or inferences, to perform computation. The associated semantics is here a computational or operational one.

\section{Methodology}

The three steps we propose consist in a semantic normalization of the terms introduced in the ontology, followed by a formalization of the meaning of the knowledge primitives obtained and an operationalization using knowledge representation languages. The two last steps are not very different from what can be found in other methodologies. The point is the way they are now integrated in a process aimed at making ontology development and use easier.

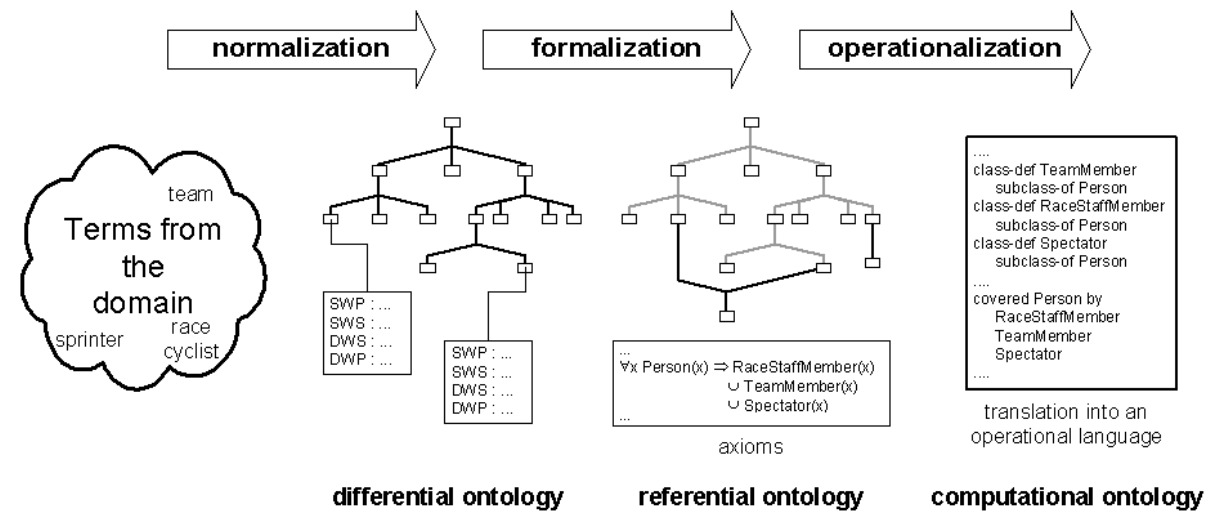

\subsection{First Step: Semantic Normalization}

The goal of the first step of this methodology is to reach a semantic agreement about the meaning of the labels used for naming the concepts. Natural language is usually the best access to the knowledge of a domain. In INA, the archivists use a collection of textual documents that are delivered with TV programs. Hence, it seems natural to look for possible labels, candidates for future primitives, within these documents.

One of our ontologies deals with the field of cycling race, especially the Tour de France event. During the analysis of that domain we discovered, for instance, numerous terms referring to human beings who do not play obviously similar roles in a cycling race : race cyclist, spectator, team manager, reporter, race supervisor, climber, wheeler, sprinter...

After having extracted labels, the ontologist has to specify their meaning clearly, and therefore to use a relevant semantic theory. We are going to build a differential ontology which will turn these terms into notions based on differential semantics ([3]). Practically, the ontologist has to be able to express the similarities and differences of each notion with respect to its neighbors: its parent-notion and its siblings-notions. The result is a taxonomy of notions, where 
the meaning of a node is given by the gathering of all similarities and differences attached to the notions found on the way from the root notion (the more generic) to this node.

We propose four principles to render explicit this information:

- The similarity with parent principle (or SWP): explicits why the notion inherits properties of the one that subsumes it;

- The similarity with siblings principle (or SWS): gives a semantic axis, a property - assuming exclusive values - allowing to compare the notion with its siblings.

- The difference with siblings principle (or DWS): precises here the property allowing to distinguish the notion from its siblings;

- The difference with parent principle (or DWP): explicits the difference allowing to distinguish the notion from its parent;

In the example given above, we can notice that terms like climber, wheeler and sprinter refer to race cyclists who are employed by teams. Actually, all the people who usually attend the Tour de France do not play the same role. We can thereby gather these terms according to the role people play during the race. Thus, the notion Person can be specialized in three new notions - Race Staff Member, Team Member and Spectator - following the differential principles given below:

\begin{tabular}{|l|}
$\longrightarrow$ For all the following notions \\
SwP: he is a person \\
Sws: a property precises why the person is present during the race \\
$\longrightarrow$ Race Staff Member \\
Dws: he is accredited by the race management \\
$\longrightarrow$ Team Member \\
DWs: he is employed by a team that takes part in the race \\
$\longrightarrow$ Spectator \\
Dws: he is neither accredited by the race management, nor employed by a team that \\
takes part in the race \\
$\longrightarrow$ For all these notions \\
DWP: $\{$ sws $+\{$ Dws
\end{tabular}

Actually, all those principles do not have the same methodological status. First, we have noticed that the $S W P$ and $S W S$ principles are shared among the notions from the same siblings. Second, the $D W P$ principle has often proved to be the sum of the principles $S W S$ and $D W S$ : we give firstly a means to create a difference, and then we put it in a concrete form to finalize the concept definition.

\subsection{Second Step: Knowledge Formalization}

The ontological tree obtained in the first step allows to disambiguate the notions and to clarify their meanings for a domain-specific application. The transition to extensional semantics aims at linking the notions to a set of referents. The 
notions become concepts behaving as formal primitives and being part of a referential ontology. Each concept refers to a set of objects in the domain (its extension). Therefore, we can use the operations that exist for sets (i.e. union, intersection or complementary) in order to obtain new concepts.

The comparison of extensions allows to define an extensional inheritance relation between concepts: one is subsumed by another if and only if its extension is included in its parent's extension. The subsumption relations of the differential ontology are still true in the referential ontology, but additional nodes may change the tree structure. For instance, Climber and wheeler are exclusive notions, but the matching formal concepts can have extensions with common individuals. Typically, the race cyclist Lance Armstrong has these two skills. Hence, we can define in the referential ontology - with a necessary and sufficient condition - a new concept ClimberAndWheeler to gather such individuals. Multiple inheritance is thereby possible.

Referential semantics allows to introduce new defined concepts but also definitions for existing concepts imported from the differential ontology. Also, the ontologist has to precise here the arity and domains of the relations. Finally, the ontologist can add some logical axioms in relation to part-whole reasoning, composition of relations, exhaustive partitions, etc. For instance, we can state in our cycling ontology that Race Staff Member, Team Member and Spectator form a disjoint coverage of the concept Person.

\subsection{Third Step: Towards a Computational Ontology}

The third and last step of the methodology allows to equip the referential concepts with the possible computational operations available in the application KBS: this is the computational ontology. The system uses an operational knowledge representation language which allows particular inferences. For a language based on the conceptual graph formalism, these inferences are graph operations (joint, projection, etc). For a language based on description logics, these inferences are mainly subsumption tests and classification.

\subsection{Implementing the Methodology: The DOE Editor}

$D O E^{6}$ (Differential Ontology Editor) is a simple prototype that supports the three steps of the methodology detailed above. It is not intended to bring a direct competition with other existing environments (like Protégé2000, OILed, OntoEdit or WebODE). Rather, its purpose is to demonstrate by experimentation how taxonomy structuring can benefit from the methodology described in this paper.

During the first step, the ontologist can enter the definition of the notions according to our principles. The tool automatizes partly this task, following the observations made in Section 3.1. The illustration below shows the interface recalling our Race Staff Member example. For the second step, it imports the

\footnotetext{
${ }^{6}$ The tool is available for free at http://opales.ina.fr/public/.
} 
taxonomies built previously and allows the ontologist to specialize existing concepts and relations, as well as to specify the arity and domains of the relations. Here the editor is able to make some consistency checking (propagation of the arity all along the hierarchy - if specified - and inheritance of domains). The last step is implemented by exporting the referential ontology into commonly-used KR languages (DAML-OIL, RDFS). This export mechanism also allows to refine the ontologies built, using the features supported by other editors.

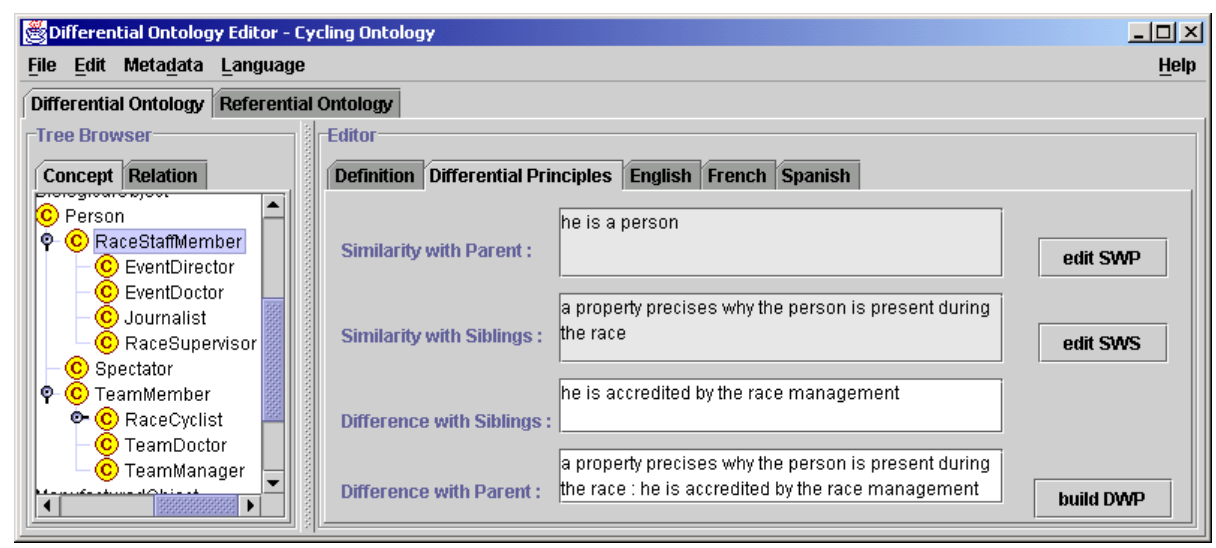

\section{Conclusion and Future Work}

In the present methodologies for building ontologies, nothing really forces the ontologist to assign a clear meaning to concepts, the comments remaining mostly informal. We have then proposed guidelines, mainly based on linguistics recommendations (using differential semantics) to explicit the linguistic meaning of the knowledge primitives of the ontology. The proposed methodology follows three steps: normalization, formalization and operationalization. We have implemented this methodology in an edition tool prototype, DOE, and several quite important ontologies have already been built within it. For the future, we plan to better integrate our solution in a more complete ontology engineering process, using for instance the results of terminological extraction tools to discover candidate-concepts and candidate-relations.

\section{References}

1. Bouaud, J., Bachimont, B., Charlet, J., Zweigenbaum, P.: Methodological principles for structuring an ontology. In IJCAI-95 Workshop on Basic Ontological Issues in Knowledge Sharing, Montreal, Canada, 1995.

2. Fernández, M., Gómez-Pérez, A. and Juristo, N.: Methontology: From Ontological Art Towards Ontological Engineering. In AAAI97 Spring Symposium Series on Ontological Engineering, 33-40, Stanford, California, 1997.

3. Rastier, F., Cavazza, M. and Abeillé, A.: Sémantique pour l'analyse. Masson, Paris, 1994. 